\title{
GENERAL RESULTS ON THE CONVERGENCE OF MULTIPOINT HERMITE-PADÉ APPROXIMANTS OF NIKISHIN SYSTEMS
}

\author{
U. FIDALGO PRIETO AND G. LÓPEZ LAGOMASINO
}

\begin{abstract}
We consider simultaneous approximation of Nikishin systems of functions by means of rational vector functions which are constructed interpolating along a prescribed table of points. We give general conditions for the uniform convergence of such approximants with a geometric rate under very weak assumptions.
\end{abstract}

\section{INTRODUCTION}

One of the basic results in rational approximation theory is Markov's Theorem (see [12]) on the convergence of diagonal Padé approximants to the Cauchy transform of a finite positive Borel measure supported on a compact subset of the real line. No restriction is imposed on the measure. In [9] the authors obtain a version of Markov's Theorem for so called multipoint Padé approximants in which the interpolation conditions are distributed along an arbitrary symmetric (with respect to the real line) triangular table of points bounded away from the convex hull of the support of the measure. The main objective of this paper is to produce a similar result for certain (Nikishin) systems of Markov functions. The approximating functions used are multipoint Hermite-Padé vector rational functions which extend the construction used in [9] to the vector case.

Let $S=\left(s_{1}, \cdots, s_{m}\right)$ be a system of finite Borel measures with constant sign, and bounded $\operatorname{support} \operatorname{supp}\left(s_{i}\right) \subset \mathbb{R}, i=1, \ldots, m$, contained in the real line consisting of infinitely many points. Let $\widehat{S}=\left(\widehat{s}_{1}, \ldots, \widehat{s}_{m}\right)$ be the corresponding system of Markov functions, where

$$
\widehat{s}_{k}(z)=\int \frac{d s_{k}(x)}{z-x}, \quad k=1, \ldots, m .
$$

We study the uniform convergence of multipoint Hermite-Padé approximants for a special type of system of Markov functions introduced by E.M. Nikishin in [13]. Let us define them.

Let $\sigma_{1}$ and $\sigma_{2}$ be two measures with constant sign supported on $\mathbb{R}$ and let $\Delta_{1}, \Delta_{2}$ denote the smallest intervals containing $\operatorname{supp}\left(\sigma_{1}\right)$ and $\operatorname{supp}\left(\sigma_{2}\right)$, respectively. We write $\Delta_{i}=\operatorname{Co}\left(\operatorname{supp}\left(\sigma_{i}\right)\right)$. Assume that $\Delta_{1} \cap \Delta_{2}=\emptyset$ and define

$$
\left\langle\sigma_{1}, \sigma_{2}\right\rangle(x)=\int \frac{d \sigma_{2}(t)}{x-t} d \sigma_{1}(x)=\widehat{\sigma}_{2}(x) d \sigma_{1}(x) .
$$

Therefore, $\left\langle\sigma_{1}, \sigma_{2}\right\rangle$ is a measure with constant sign and support equal to that of $\sigma_{1}$.

Definition 1. For a system of intervals $\Delta_{1}, \ldots, \Delta_{m}$ contained in $\mathbb{R}$ satisfying $\Delta_{j} \cap \Delta_{j+1}=\emptyset$, $j=1, \ldots, m-1$, and finite Borel measures $\sigma_{1}, \ldots, \sigma_{m}$ with constant sign in $\operatorname{Co}\left(\operatorname{supp}\left(\sigma_{j}\right)\right)=\Delta_{j}$ and such that, each one has infinitely many points in its support, we define by induction

$$
\left\langle\sigma_{1}, \sigma_{2}, \ldots, \sigma_{j}\right\rangle=\left\langle\sigma_{1},\left\langle\sigma_{2}, \ldots, \sigma_{j}\right\rangle\right\rangle, \quad j=2, \ldots, m .
$$

We say that $S=\left(s_{1}, \ldots, s_{m}\right)=\mathcal{N}\left(\sigma_{1}, \ldots, \sigma_{m}\right)$, where

$$
s_{1}=\left\langle\sigma_{1}\right\rangle=\sigma_{1}, \quad s_{2}=\left\langle\sigma_{1}, \sigma_{2}\right\rangle, \ldots, s_{m}=\left\langle\sigma_{1}, \ldots, \sigma_{m}\right\rangle
$$

is the Nikishin system of measures generated by $\left(\sigma_{1}, \ldots, \sigma_{m}\right)$.

1991 Mathematics Subject Classification. Primary 30E10, 42C05.

The work of both authors was supported by Ministerio de Ciencia y Tecnología under grant BFM 2003-06335C03-02. The second author was also partially supported by NATO PST.CLG.979738 and INTAS 03-51-6637. 
Notice that all the measures in a Nikishin system have the same support, namely $\operatorname{supp}\left(\sigma_{1}\right)$. Take an arbitrary Nikishin system of measures $S=\left(s_{1}, \ldots, s_{m}\right)$, and let $\widehat{S}=\left(\widehat{s}_{1}, \ldots, \widehat{s}_{m}\right)$ be the corresponding Nikishin system of functions. We fix a multi-index $\mathbf{n}=\left(n_{1}, \ldots, n_{m}\right) \in \mathbb{Z}_{+}^{m}$ and denote $|\mathbf{n}|=n_{1}+\cdots+n_{m}$. Let $\alpha_{\mathbf{n}}$ be a monic polynomial with real coefficients, whose zeros lie in $D=\overline{\mathbb{C}} \backslash \Delta_{1}$, and $\operatorname{deg} \alpha_{\mathbf{n}} \leq|\mathbf{n}|+\min \left\{n_{i}\right\}$. It is easy to verify that there exist polynomials $Q_{\mathbf{n}}$, $P_{\mathbf{n}, i}, i=1, \ldots m$, such that

$$
\begin{aligned}
& \text { i) } \quad \operatorname{deg} Q_{\mathbf{n}} \leq|\mathbf{n}|, \quad Q_{\mathbf{n}} \neq 0, \quad \operatorname{deg} P_{\mathbf{n}, i} \leq|\mathbf{n}|-1 \\
& \text { ii) } \quad\left[\frac{Q_{\mathbf{n}} \widehat{s}_{i}-P_{\mathbf{n}, i}}{\alpha_{\mathbf{n}}}\right](z)=\mathcal{O}\left(\frac{1}{z^{n+1}}\right) \in H(D), \quad i=1, \ldots, m .
\end{aligned}
$$

Finding $Q_{\mathbf{n}}, P_{\mathbf{n}, i}, i=1, \ldots, m$, reduces to solving a system of $(m+1)|\mathbf{n}|$ homogeneous linear equations on the $(m+1)|\mathbf{n}|+1$ unknown coefficients of the polynomials. Thus, a nontrivial solution exists. $P_{\mathbf{n}, i}$ interpolates the function $Q_{\mathbf{n}} \widehat{s}_{i}$ at the zeros of $\alpha_{\mathbf{n}}$. We call $R_{\mathbf{n}}=\left(R_{\mathbf{n}, 1}, \ldots, R_{\mathbf{n}, m}\right), R_{\mathbf{n}, i}=$ $\frac{P_{\mathbf{n}, i}}{Q_{\mathbf{n}}}, i=1, \ldots, m$, a multi-point Hermite-Padé approximant of the system of functions $\widehat{S}$, associated to the multi-index $\mathbf{n}$ and the polynomial $\alpha_{\mathbf{n}}$. If $\alpha_{\mathbf{n}} \equiv 1$, we have a classical Hermite-Padé approximant.

We study the uniform convergence of the sequences $\left\{R_{\mathbf{n}, i}\right\}_{\mathbf{n} \in \Lambda}, \Lambda \subset \mathbb{Z}_{+}^{m}, i=1, \ldots, m$, to the functions $\widehat{s}_{i}$ respectively. First, we obtain convergence in a weaker sense for a wide class of sequences of multi-indices $\Lambda$. Then, restricting a bit more the type of multi-indices considered, we obtain uniform convergence on each compact subset of $\overline{\mathbb{C}} \backslash \Delta_{1}$.

Let us introduce the type of weak convergence to be considered. Let $E \subset \mathbb{C}$ and $\mathcal{F}$ be the set of all open coverings $\left\{U_{\nu}\right\}$ of $E$ by disks $U_{\nu}$. Let $\left|U_{\nu}\right|$ denote the radius of $U_{\nu}$. We define

$$
m_{1}(E)=\inf \left\{\sum\left|U_{\nu}\right|:\left\{U_{\nu}\right\} \in \mathcal{F}\right\}
$$

to be the 1 dimensional Hausdorff content of $E$. From the definition it is easy to prove that the Hausdorff content is sub-additive and monotonic. More on this concept and its properties may be found in [11].

Let $f_{n}, n \in \mathbb{N}$, and $f$ be complex-valued functions defined on a region $\Omega \subset \mathbb{C}$. We write

$$
\mathcal{H}-\lim _{n \rightarrow \infty} f_{n}=f, \quad K \subset \Omega,
$$

and say that $f_{n}$ converges in 1-Hausdorff content to $f$ on each compact subset $K$ of $\Omega$ if for each $K \subset \Omega$ and $\epsilon>0$

$$
\lim _{n \rightarrow \infty} m_{1}\left(\left\{z \in K:\left|\left(f_{n}-f\right)(z)\right| \geq \epsilon\right\}\right)=0 .
$$

In the sequel $\Lambda \subset \mathbb{Z}_{+}^{m}$ is a sequence of distinct multi-indices, and $\left\{\alpha_{\mathbf{n}}\right\}, \mathbf{n} \in \Lambda$, a sequence of polynomials with real coefficients whose zeros lie in a compact subset $F \subset D=\overline{\mathbb{C}} \backslash \Delta_{1}$. Let $\varphi_{t}, t \in \overline{\mathbb{C}} \backslash \Delta_{1}$, denote the conformal mapping from $D$ onto the unit disk, such that $\varphi_{t}(t)=0$ and $\varphi_{t}^{\prime}(t)>0$. We have

ThEOREM 1. Let $S=\left(s_{1}, \ldots, s_{m}\right)=\mathcal{N}\left(\sigma_{1}, \ldots, \sigma_{m}\right)$ be a Nikishin system and $\Lambda \subset \mathbb{Z}_{+}^{m}$. We assume that there exists a positive constant $c$ such that for all $\mathbf{n} \in \Lambda$ and $i=1, \ldots, m$

$$
n_{i} \geq \frac{|\mathbf{n}|}{m}-c|\mathbf{n}|^{\kappa}, \quad \kappa<1 .
$$

Then, for each compact set $K \subset \mathbb{C} \backslash \Delta_{1}, \varepsilon>0$, and each $i=1, \ldots, m$, we have

$$
\limsup _{\mathbf{n} \in \Lambda}\left(m_{1}\left(\left\{z \in K:\left|\left(\widehat{s}_{i}-R_{\mathbf{n}, i}\right)(z)\right|>\varepsilon\right\}\right)\right)^{1 / 2|\mathbf{n}|} \leq \delta_{K}<1,
$$

where

$$
\delta_{K}=\max \left\{\left|\varphi_{t}(z)\right|: z \in K, t \in F \cup \Delta_{2} \cup\{\infty\}\right\} .
$$

In particular,

$$
\mathcal{H}-\lim _{\mathbf{n} \in \Lambda} R_{\mathbf{n}, i}=\widehat{s}_{i}, \quad K \subset \mathbb{C} \backslash \Delta_{1}, \quad i=1, \ldots, m
$$


If the rational functions $R_{\mathbf{n}, i}$ are holomorphic in $D$, one can use a lemma of A. A. Gonchar (see [8, Lemma 1]) which guarantees that then convergence in 1-Hausdorff content yields uniform convergence on each compact subset of $D$. When $m=1$ we have multipoint Padé approximants and it is well known that $Q_{n}, n \in \mathbb{Z}_{+}$, has $n$ simple zeros that lie in $\Delta_{1}$. The corresponding statement was proved in [3] for all $Q_{\mathbf{n}}, \mathbf{n} \in \mathbb{Z}_{+}^{2}$. In [5], the authors prove that $Q_{\mathbf{n}}$ has $|\mathbf{n}|$ simple zeros that lie on $\Delta_{1}$ for all $\mathbf{n} \in \mathbb{Z}_{+}^{3}$. The question of whether or not this remains true when $m>3$ remains open. For general $m \in \mathbb{Z}_{+}, m \geq 4$, the best known result says (see [6]): Let

$$
\mathbb{Z}_{+}^{m}(*)=\left\{\mathbf{n} \in \mathbb{Z}_{+}^{m} \mid \nexists 1 \leq i<j<k \leq m: n_{i}<n_{j}<n_{k}\right\} .
$$

Then, $Q_{\mathbf{n}}$ has $|\mathbf{n}|$ simple zeros that lie on $\Delta_{1}$ for all $\mathbf{n} \in \mathbb{Z}_{+}^{m}(*)$.

Taking into consideration the previous remarks, from Theorem 1 we immediately obtain

COROLlary 1. Under the assumptions of Theorem 1, if $m \leq 3$ or $m>3$ and $\Lambda \subset \mathbb{Z}_{+}^{m}(*)$, we have that for each compact set $K \subset D$

$$
\limsup _{\mathbf{n} \in \Lambda}\left\|\widehat{s}_{i}-R_{\mathbf{n}, i}\right\|_{K}^{1 / 2|\mathbf{n}|} \leq \delta_{K}<1, \quad i=1, \ldots, m .
$$

and

$$
\lim _{\mathbf{n} \in \Lambda} R_{\mathbf{n}, i}=\widehat{s}_{i}, \quad i=1, \ldots, m,
$$

uniformly on each compact subset $K$ of $D$.

In [7] (see Theorem 7 and the remark at the end of section 5) we obtained the exact rate of convergence in terms of the solution of a vector valued equilibrium problem in the presence of an external field. There, the measures are assumed to satisfy $\sigma_{i} \in \mathbf{R e g}, i=1, \ldots, m$, in the sense defined in [14], and the sequence $\left|\alpha_{\mathbf{n}}\right|^{1 /|\mathbf{n}|}, \mathbf{n} \in \Lambda$, is required to have limit. As compared to the results in [7], Theorem 1 and Corollary 1 are less precise in the expression of the rate of convergence but much more general in its range of application. Taking $\kappa=0$ in Theorem 1 and Corollary 1, we obtain the main results of [1] (for the case when the measures have compact supports).

Section 2 contains some lemmas. The last section is dedicated to the proof of Theorem 1 . We end that section with two remarks on extensions.

\section{Auxiliary Results}

From ii), we have

$$
z^{\nu}\left[\frac{Q_{\mathbf{n}} \widehat{s}_{i}-P_{\mathbf{n}, i}}{\alpha_{\mathbf{n}}}\right](z)=\mathcal{O}\left(\frac{1}{z^{2}}\right) \in H(D), \quad \nu=0, \ldots, n_{i}-1, \quad i=1, \ldots m .
$$

Let $\Gamma$ be a closed integration path with wilding number 1 for all its interior points. We denote by $\operatorname{Int}(\Gamma)$ and $\operatorname{Ext}(\Gamma)$ the bounded and unbounded connected components, respectively, of the complement of $\Gamma$. Take $\Gamma$ so that the zeros of $\alpha_{\mathbf{n}}$ lie in $\operatorname{Ext}(\Gamma)$ and $\operatorname{supp}\left(s_{i}\right) \subset \operatorname{Int}(\Gamma)$. Using Cauchy's Theorem

$$
0=\int_{\Gamma} z^{\nu}\left[\frac{Q_{\mathbf{n}} \widehat{s}_{i}-P_{\mathbf{n}, i}}{\alpha_{\mathbf{n}}}\right](z) d z=\int_{\Gamma} z^{\nu} \frac{Q_{\mathbf{n}} \widehat{s}_{i}}{\alpha_{\mathbf{n}}}(z) d z, \quad \nu=0, \ldots, n_{i}-1 .
$$

Taking into account (1), Fubini's Theorem, and Cauchy's Integral Formula, we deduce that $Q_{\mathbf{n}}$ satisfies the following multi-orthogonal conditions with respect to the measures of the system $S$

$$
0=\int x^{\nu} Q_{\mathbf{n}}(x) \frac{d s_{i}(x)}{\alpha_{\mathbf{n}}(x)}, \quad \nu=0, \ldots, n_{i}-1, \quad i=1, \ldots, m .
$$

Definition 2. Let $\mathbf{n}=\left(n_{1}, \ldots, n_{m}\right) \in \mathbb{Z}_{+}^{m}$. For each $i=1, \ldots, m$, we define an associated multi-index $\mathbf{n}^{i}=\left(n_{2}^{i}, \ldots, n_{m}^{i}\right)$ whose $m-1$ components satisfy

$$
n_{j}^{i}=\left\{\begin{array}{lll}
\min \left(n_{1}, \ldots, n_{j-1}, n_{i}-1\right), & \text { when } \quad j=2, \ldots, i, \\
\min \left(n_{i}, n_{j}\right), & \text { when } \quad j=i+1, \ldots, m .
\end{array}\right.
$$

We denote $\left|\mathbf{n}^{i}\right|=\sum_{j=2}^{m} n_{j}^{i}$. 
Let $1 \leq i<j \leq m$. Set

$$
s_{i, j}=\left\langle\sigma_{i}, \ldots, \sigma_{j}\right\rangle \quad\left(s_{j, j}=\sigma_{j}\right) .
$$

It is well known (see the appendix in [10]) that there exists a first degree polynomial $\mathcal{L}_{i, j}$ and a finite positive Borel measure $\tau_{i, j}, \operatorname{Co}\left(\operatorname{supp}\left(\tau_{i, j}\right)\right) \subset \operatorname{Co}\left(\operatorname{supp}\left(s_{i, j}\right)\right)$ such that

$$
\frac{1}{\widehat{s}_{i, j}(z)}=\mathcal{L}_{i, j}(z)+\widehat{\tau}_{i, j}(z) .
$$

For each $k \in\{1, \ldots, m\}$ we define an auxiliary Nikishin system $S^{k}=\left(s_{2}^{k}, \ldots, s_{m}^{k}\right)=\mathcal{N}\left(\sigma_{2}^{k}, \ldots, \sigma_{m}^{k}\right)$. If $k=1$, we take

$$
S^{1}=\left(s_{2}^{1}, \ldots, s_{m}^{1}\right)=\left(d \sigma_{2}, w_{3}^{1} d \sigma_{2}, \ldots, w_{m}^{1} d \sigma_{2}\right)=\mathcal{N}\left(\sigma_{2}, \ldots \sigma_{m}\right) .
$$

When $2 \leq k \leq m$, then

$$
\begin{gathered}
S^{k}=\left(s_{2}^{k}, \ldots, s_{m}^{k}\right)=\left(\tau_{2, k}, w_{3}^{k} d \tau_{2, k}, \ldots, w_{m}^{k} d \tau_{2, k}\right)= \\
\mathcal{N}\left(\tau_{2, k}, \widehat{s}_{2, k} d \tau_{3, k}, \ldots, \widehat{s}_{k-1, k} d \tau_{k, k}, \widehat{s}_{k, k} d \sigma_{k+1}, \sigma_{k+2}, \ldots, \sigma_{m}\right) .
\end{gathered}
$$

These auxiliary systems were introduced in [1]. Subsequently, in Theorem 3.1.3 of [4] certain relations between Markov functions corresponding to different components of distinct auxiliary Nikishin systems were established. For convenience of the reader, we write these formulas according to our notation. We have:

$$
\begin{gathered}
\frac{1}{\widehat{s}_{k}^{1}(z)}=\mathcal{L}_{k}(z)+\widehat{s}_{2}^{k}(z), \\
\frac{\widehat{s}_{j}^{1}(z)}{\widehat{s}_{k}^{1}(z)}=a_{j}+\widehat{s}_{j+1}^{k}(z)+c_{j} \widehat{s}_{j}^{k}(z), \quad j=2, \ldots, k-1,
\end{gathered}
$$

and

$$
\frac{\widehat{s}_{j}^{1}(z)}{\widehat{s}_{k}^{1}(z)}=a_{j}+\widehat{s}_{j}^{k}(z), \quad j=k+1, \ldots, m,
$$

where $a_{j}$ and $c_{j}$ denote constants and $\mathcal{L}_{k}$ is a polynomial of degree 1 .

Set

$$
\Phi_{\mathbf{n}, i}(z)=\left[\frac{Q_{\mathbf{n}} \widehat{s}_{i}-P_{\mathbf{n}, i}}{\alpha_{\mathbf{n}}}\right](z) .
$$

Given $Q_{\mathbf{n}}$ satisfying ii), for each fixed $i=1, \ldots, m, P_{\mathbf{n}, i}$ is uniquely determined by the formula

$$
P_{\mathbf{n}, i}(z)=\int \frac{Q_{\mathbf{n}}(z) \alpha_{\mathbf{n}}(x)-Q_{\mathbf{n}}(x) \alpha_{\mathbf{n}}(z)}{z-x} \frac{d s_{i}(x)}{\alpha_{\mathbf{n}}(x)} .
$$

In fact, it is easy to see that the expression defined by the integral is a polynomial of degree $\leq|\mathbf{n}|-1$ since it equals

$$
\int\left(\frac{Q_{\mathbf{n}}(z)-Q_{\mathbf{n}}(x)}{z-x} \alpha_{\mathbf{n}}(x)-\frac{\alpha_{\mathbf{n}}(z)-\alpha_{\mathbf{n}}(x)}{z-x} Q_{\mathbf{n}}(x)\right) \frac{d s_{i}(x)}{\alpha_{\mathbf{n}}(x)} .
$$

Let $P_{\mathbf{n}, i}^{\prime}$ be the polynomial defined by (12). This formula may be rewritten as

$$
\left[\frac{Q_{\mathbf{n}} \widehat{s}_{i}-P_{\mathbf{n}, i}^{\prime}}{\alpha_{\mathbf{n}}}\right](z)=\int \frac{Q_{\mathbf{n}}(x)}{z-x} \frac{d s_{i}(x)}{\alpha_{\mathbf{n}}(x)}=\mathcal{O}\left(\frac{1}{z}\right) \in H(D) .
$$

If $P_{\mathbf{n}, i}$ comes from ii), subtracting ii) from the previous relation it follows that

$$
\left[\frac{P_{\mathbf{n}, i}-P_{\mathbf{n}, i}^{\prime}}{\alpha_{\mathbf{n}}}\right](z)=\mathcal{O}\left(\frac{1}{z}\right) \in H(D) .
$$

Consequently, $P_{\mathbf{n}, i} \equiv P_{\mathbf{n}, i}^{\prime}$ as stated.

Lemma 1. Let $\mathbf{n}=\left(n_{1}, \ldots, n_{m}\right)$ be a multi-index. For each $i=1, \ldots m$, we have

$$
\int_{\Delta_{2}}\left(h_{j} \Phi_{\mathbf{n}, i}\right)\left(x_{2}\right) d s_{j}^{i}\left(x_{2}\right)=0, \quad j=2, \ldots, m, \quad \operatorname{deg} h_{j}<n_{j}^{i} .
$$


Proof. Fix $i \in\{1, \ldots, m\}$. From the definition of $\Phi_{\mathbf{n}, i}$ and (13), we obtain

$$
\int_{\Delta_{2}}\left(h_{j} \Phi_{\mathbf{n}, i}\right)\left(x_{2}\right) d s_{j}^{i}\left(x_{2}\right)=\int_{\Delta_{2}} h_{j}\left(x_{2}\right) \int_{\Delta_{1}} \frac{Q_{\mathbf{n}}\left(x_{1}\right)}{x_{2}-x_{1}} \frac{d s_{i}\left(x_{1}\right)}{\alpha_{\mathbf{n}}\left(x_{1}\right)} d s_{j}^{i}\left(x_{2}\right) .
$$

Since $\operatorname{deg} h_{j}<n_{j}^{i} \leq n_{i}$, from (8) and Fubini's Theorem, it follows that

$$
\begin{gathered}
\int_{\Delta_{2}} h_{j}\left(x_{2}\right) \int_{\Delta_{1}} \frac{Q_{\mathbf{n}}\left(x_{1}\right)}{x_{2}-x_{1}} \frac{d s_{i}\left(x_{1}\right)}{\alpha_{\mathbf{n}}\left(x_{1}\right)} d s_{j}^{i}\left(x_{2}\right)=\int_{\Delta_{2}} \int_{\Delta_{1}} \frac{\left(h_{j} Q_{\mathbf{n}}\right)\left(x_{1}\right)}{x_{2}-x_{1}} \frac{d s_{i}\left(x_{1}\right)}{\alpha_{\mathbf{n}}\left(x_{1}\right)} d s_{j}^{i}\left(x_{2}\right)= \\
=-\int_{\Delta_{1}}\left(h_{j} Q_{\mathbf{n}} \widehat{s}_{j}^{i}\right)\left(x_{1}\right) \frac{d s_{i}\left(x_{1}\right)}{\alpha_{\mathbf{n}}\left(x_{1}\right)} .
\end{gathered}
$$

First, we prove the statement of the lemma for the case $i+1 \leq j \leq m$. If $i=m$, the set of $j$ is empty and there is nothing to prove. Let $i \leq m-1$. Using (11), we obtain that

$$
\begin{gathered}
-\int_{\Delta_{1}}\left(h_{j} Q_{\mathbf{n}} \widehat{s}_{j}^{i}\right)\left(x_{1}\right) \frac{d s_{i}\left(x_{1}\right)}{\alpha_{\mathbf{n}}\left(x_{1}\right)}=\int_{\Delta_{1}}\left(h_{j} Q_{\mathbf{n}}\right)\left(x_{1}\right)\left(\frac{\widehat{s}_{j}^{1}\left(x_{1}\right)}{\widehat{s}_{i}^{1}\left(x_{1}\right)}-a_{j}\right) \frac{d s_{i}\left(x_{1}\right)}{\alpha_{\mathbf{n}}\left(x_{1}\right)}= \\
-a_{j} \int_{\Delta_{1}}\left(h_{j} Q_{\mathbf{n}}\right)\left(x_{1}\right) \frac{d s_{i}\left(x_{1}\right)}{\alpha_{\mathbf{n}}\left(x_{1}\right)}+\int_{\Delta_{1}}\left(h_{j} Q_{\mathbf{n}} \widehat{s}_{j}^{1}\right)\left(x_{1}\right) \frac{d \sigma_{1}\left(x_{1}\right)}{\alpha_{\mathbf{n}}\left(x_{1}\right)} .
\end{gathered}
$$

In the last equality we have the sum of two terms. By hypothesis $\operatorname{deg} h_{j}<n_{j}^{i} \leq \min \left\{n_{i}, n_{j}\right\}$. Taking into account (8), we deduce that both terms vanish. Hence the first case is proved.

Now, we analyze the case when $2 \leq j \leq i$. Using several times formula (10) to make $j$ descend to 2 and finally formula (9), we obtain the equalities

$$
\begin{gathered}
\widehat{s}_{j}^{i}=\frac{\widehat{s}_{j-1}^{1}}{\widehat{s}_{i}^{1}}-a_{j-1}-c_{j-1} \widehat{s}_{j-1}^{i}= \\
\frac{\widehat{s}_{j-1}^{1}}{\widehat{s}_{i}^{1}}-a_{j-1}-c_{j-1}\left(\frac{\widehat{s}_{j-2}^{1}}{\widehat{s}_{i}^{1}}-a_{j-2}-c_{j-2} \widehat{s}_{j-2}^{1}\right)=\cdots=\mathcal{L}_{i}+\frac{1}{\widehat{s}_{i}^{1}} \sum_{k=1}^{j-1} c_{k-1} \widehat{s}_{k}^{1},
\end{gathered}
$$

where $\mathcal{L}_{i}$ denotes a polynomial of degree $1, \widehat{s}_{1}^{i} \equiv 1$, and $c_{k-1}, k=2, \ldots, j-1$, are constants. Sustituting (16) into (15), we have that

$$
\begin{gathered}
\int_{\Delta_{1}}\left(h_{j} Q_{\mathbf{n}}\right)\left(x_{1}\right) \frac{d s_{i}\left(x_{1}\right)}{\alpha_{\mathbf{n}}\left(x_{1}\right)}= \\
=-\int_{\Delta_{1}}\left(h_{j} Q_{\mathbf{n}}\right)\left(x_{1}\right) \mathcal{L}_{i}\left(x_{1}\right) \frac{d s_{i}\left(x_{1}\right)}{\alpha_{\mathbf{n}}\left(x_{1}\right)}-\sum_{k=1}^{j-1} c_{k-1} \int_{\Delta_{1}}\left(h_{j} Q_{\mathbf{n}}\right)\left(x_{1}\right) \frac{d s_{k}\left(x_{1}\right)}{\alpha_{\mathbf{n}}\left(x_{1}\right)} .
\end{gathered}
$$

From (8) and the hypothesis $\operatorname{deg} h_{j} \leq \min \left(n_{1}-1, \ldots, n_{j-1}-1, n_{i}-2\right)$, we obtain that all the integrals on the right hand side of this equality are zero. Hence, we have finished the proof.

In the proof of Theorem 2 below, we use the concept of AT system introduced by E. M. Nikishin in $[13]$.

DEFINITION 3. Let $\omega_{i}, i=1, \ldots, m$, be a continuous functions with constant sign on an interval $[a, b]$ of the real line. It is said that $\left(\omega_{1}, \ldots, \omega_{m}\right)$ forms an AT system for the index $\mathbf{n}=\left(n_{1}, \ldots, n_{m}\right)$ on $[a, b]$ if no matter what polynomials $h_{1}, \ldots, h_{m}$ one chooses with $\operatorname{deg} h_{i} \leq n_{i}-1, i=1, \ldots, m$, not all identically equal to zero, the function

$$
\mathcal{H}_{\mathbf{n}}(x)=\mathcal{H}_{\mathbf{n}}\left(h_{1}, \ldots, h_{m} ; x\right)=h_{1}(x) \omega_{1}(x)+\cdots+h_{m}(x) \omega_{m}(x)
$$

has at most $|\mathbf{n}|-1$ zeros on $[a, b]\left(\operatorname{deg} h_{j} \leq-1\right.$ forces $\left.h_{i} \equiv 0\right)$.

Regarding AT systems, Theorem 2 of [5] and Theorem 2 of [6] may be jointly stated as follows.

LEMMA 2. Let $\left(s_{2}, \ldots, s_{m}\right)=\mathcal{N}\left(\sigma_{2}, \ldots, \sigma_{m}\right)$ be an arbitrary Nikishin system of $m-1$ measures. If $m \in\{2,3\}$ the system of functions $\left(1, \widehat{s}_{2}, \ldots, \widehat{s}_{m}\right)$ forms an AT system for any index $\mathbf{n} \in \mathbb{Z}_{+}^{m}$ on any interval $[a, b]$ disjoint from $\Delta_{2}$. When $m \geq 4$ the system of functions $\left(1, \widehat{s}_{2}, \ldots, \widehat{s}_{m}\right)$ forms an $A T$ system for any index $\mathbf{n} \in \mathbb{Z}_{+}^{m}(*)$ on any interval $[a, b]$ disjoint from $\Delta_{2}$. 
The observations made after the statement of Theorem 1 that when $m \leq 3$, or $m>3$ and $\mathbf{n} \in \mathbb{Z}_{+}^{m}(*)$, all $Q_{\mathbf{n}}$ have exactly $|\mathbf{n}|$ simple zeros in $\Delta_{1}$ is an immediate consequence of Lemma 2 (see [5] and [6]).

Theorem 2. Let $S=\left(s_{1}, \ldots, s_{m}\right)=\mathcal{N}\left(\sigma_{1}, \ldots, \sigma_{m}\right)$ be a Nikishin system and $\mathbf{n} \in \mathbb{Z}_{+}^{m}$. For each $i=1, \ldots, m$, set $N^{i}=\left|\mathbf{n}^{i}\right|+n_{i}$, where $\mathbf{n}^{i}$ is the associated multi-index. Then, for each $i=1, \ldots, m$, there exists a monic polynomial $W_{\mathbf{n}, i}, \operatorname{deg} W_{\mathbf{n}, i} \geq\left|\mathbf{n}^{i}\right|$, whose zeros are simple and lie in the interior of $\operatorname{Co}\left(\operatorname{supp}\left(\sigma_{2}\right)\right)$, such that

$$
0=\int x^{\nu} Q_{\mathbf{n}}(x) \frac{d s_{i}(x)}{\left(\alpha_{\mathbf{n}} W_{\mathbf{n}, i}\right)(x)}, \quad \nu=0,1, \ldots, N^{i}-1 .
$$

and

$$
\left[\frac{Q_{\mathbf{n}} \widehat{s}_{i}-P_{\mathbf{n}, i}}{\alpha_{\mathbf{n}} W_{\mathbf{n}, i}}\right](z)=\frac{1}{q(z)} \int \frac{\left(q Q_{\mathbf{n}}\right)(x)}{\left(\alpha_{\mathbf{n}} W_{\mathbf{n}, i}\right)(x)} \frac{d s_{i}(x)}{z-x}=\mathcal{O}\left(\frac{1}{z^{N^{i}+1}}\right) \in H(D),
$$

where $q$ is an arbitrary polynomial with degree $\leq N^{i}$.

Proof. Proving the existence of the polynomial $W_{\mathbf{n}, i}$ is equivalent to showing that the function $\Phi_{\mathbf{n}, i}=\left[\left(Q_{\mathbf{n}} \widehat{s}_{i}-P_{\mathbf{n}, i}\right) / \alpha_{\mathbf{n}}\right]$ has at least $\left|\mathbf{n}^{i}\right|$ changes of sign in the interior of $\operatorname{Co}\left(\operatorname{supp}\left(\sigma_{2}\right)\right)$.

Consider the system of functions $\left(\widehat{s}_{3,2}^{i}, \ldots, \widehat{s}_{3, m}^{i}\right)$, where $\widehat{s}_{3,2}^{i} \equiv 1$ and for each $j=3, \ldots, m, \widehat{s}_{3, j}^{i}$ is the Markov function of the measure $s_{3, j}^{i}=\left\langle\sigma_{3}^{i}, \ldots, \sigma_{j}^{i}\right\rangle$. Notice that $d s_{j}^{i}=\widehat{s}_{3, j}^{i} d \sigma_{2}^{i}, j=2, \ldots, m$. Rewriting with this notation the equality (14) of Lemma 1, we have

$$
\int_{\Delta_{2}}\left(h_{j} \widehat{s}_{3, j}^{i} \Phi_{\mathbf{n}, i}\right)(x) d \sigma_{2}^{i}=0, \quad j=2, \ldots, m,
$$

where for each $j=2, \ldots, m, h_{j}$ is an arbitrary polynomial of degree $<n_{j}^{i}$. Hence

$$
\int_{\Delta_{2}}\left(\mathcal{H} \Phi_{\mathbf{n}, i}\right)(x) d \sigma_{2}^{i}=0
$$

with

$$
\mathcal{H}(x)=\mathcal{H}\left(h_{2}, \ldots, h_{m} ; x\right)=\sum_{j=2}^{m}\left(h_{j} \widehat{s}_{3, j}^{i}\right)(x), \quad \operatorname{deg} h_{j}<n_{j}^{i} .
$$

Let us assume that $\Phi_{\mathbf{n}, i}$ has at most $N$ changes of sign in the interior of the interval $\Delta_{2}$, where $N \leq\left|\mathbf{n}^{i}\right|-1$. Then, we can choose polynomials $h_{j}, j=2, \ldots, m$, such that the function $\mathcal{H}\left(h_{2}, \ldots, h_{m}, x\right)$ has a simple zero at each point where $\Phi_{\mathbf{n}, i}$ changes sign in the interior of $\Delta_{2}$ and a zero of multiplicity $\left|\mathbf{n}^{i}\right|-N-1$ at one of the extreme points of $\Delta_{2}$. According to Lemma 2 , the system of functions $\left(1, \widehat{s}_{3,3}^{i}, \ldots, \widehat{s}_{3, m}^{i}\right)$ forms an AT system on $\Delta_{2}$. Consequently, $\mathcal{H}$ cannot have more zeros on $\Delta_{2}$ than those that we have assigned and $\mathcal{H} \Phi_{\mathbf{n}, i}$ has constant sign on $\Delta_{2}$. This contradicts (20). Therefore, $\Phi_{\mathbf{n}, i}$ has at least $\left|\mathbf{n}^{i}\right|$ changes of sign in the interior of $\Delta_{2}$. Let $W_{\mathbf{n}, i}$ be the monic polynomial whose zeros lie at the points where $\Phi_{\mathbf{n}, i}$ changes of sign in the interior of $\Delta_{2}$. From ii) in (2) and taking into account that $\operatorname{deg} W_{\mathbf{n}, i} \geq\left|\mathbf{n}^{i}\right|$ we obtain that

$$
\left[\frac{Q_{\mathbf{n}} \widehat{s}_{i}-P_{\mathbf{n}, i}}{\alpha_{\mathbf{n}} W_{\mathbf{n}, i}}\right](z)=\mathcal{O}\left(\frac{1}{z^{N^{i}+1}}\right) \in H(D) .
$$

Now, let us prove (18). Let $\Gamma$ be a closed integration path with winding number 1 for all its interior points, such that the zeros of $\alpha_{\mathbf{n}}$ and the interval $\Delta_{2}$ are in $\operatorname{Ext}(\Gamma)$ and $\Delta_{1} \subset \operatorname{Int}(\Gamma)$. From (21) and Cauchy's Theorem, we obtain for each $i=1, \ldots, m$,

$$
0=\int_{\Gamma} z^{\nu}\left[\frac{Q_{\mathbf{n}} \widehat{s}_{i}-P_{\mathbf{n}, i}}{\alpha_{\mathbf{n}} W_{\mathbf{n}, i}}\right](z) d z, \quad \nu=0, \ldots, N^{i}-1 .
$$

Substituting $\widehat{s}_{i}$ by its integral expression, we have that

$$
0=\int_{\Gamma} z^{\nu}\left[\frac{Q_{\mathbf{n}}}{\alpha_{\mathbf{n}} W_{\mathbf{n}, i}}\right](z) \int_{\Delta_{2}} \frac{d s_{i}(x)}{z-x} d z-\int_{\Gamma} z^{\nu}\left[\frac{P_{\mathbf{n}, i}}{\alpha_{\mathbf{n}} W_{\mathbf{n}, i}}\right](z) d z, \quad \nu=0, \ldots, N^{i}-1 .
$$

On the right hand side, the second term vanishes since $P_{\mathbf{n}, i} /\left(\alpha_{\mathbf{n}} W_{\mathbf{n}, i}\right)$ is holomorphic in $\operatorname{Int}(\Gamma)$. Using Fubini's Theorem and Cauchy's Integral Formula, we obtain (18). 
Assume that $\operatorname{deg} q \leq N^{i}$. From (18), we have that

$$
0=\int_{\Delta_{1}} \frac{q(z)-q(x)}{z-x} Q_{\mathbf{n}}(x) \frac{d s_{i}(x)}{\alpha_{\mathbf{n}} W_{\mathbf{n}, i}(x)} .
$$

Hence,

$$
\int_{\Delta_{1}} \frac{Q_{\mathbf{n}}(x)}{W_{\mathbf{n}, i}(x)} \frac{d s_{i}(x)}{(z-x) \alpha_{\mathbf{n}}(x)}=\frac{1}{q(z)} \int_{\Delta_{1}} \frac{\left(q Q_{\mathbf{n}}\right)(x)}{W_{\mathbf{n}, i}(x)} \frac{d s_{i}(x)}{(z-x) \alpha_{\mathbf{n}}(x)}
$$

Define

$$
\widehat{P}_{i}(z)=\int_{\Delta_{1}} \frac{Q_{\mathbf{n}}(z)\left(\alpha_{\mathbf{n}} W_{\mathbf{n}, i}\right)(x)-Q_{\mathbf{n}}(x)\left(\alpha_{\mathbf{n}} W_{\mathbf{n}, i}\right)(z)}{z-x} \frac{d s_{i}(x)}{\left(\alpha_{\mathbf{n}} W_{\mathbf{n}, i}\right)(x)}
$$

The function $\widehat{P}_{i}$ is a polynomial of degree $\leq|\mathbf{n}|-1$ because

$$
\widehat{P}_{\mathbf{n}, i}(z)=\int_{\Delta_{1}}\left[\frac{Q_{\mathbf{n}}(z)-Q_{\mathbf{n}}(x)}{z-x}\left(\alpha_{\mathbf{n}} W_{\mathbf{n}, i}\right)(x)-\frac{\left(\alpha_{\mathbf{n}} W_{\mathbf{n}, i}\right)(z)-\left(\alpha_{\mathbf{n}} W_{\mathbf{n}, i}\right)(x)}{z-x} Q_{\mathbf{n}}(x)\right] \frac{d s_{i}(x)}{\left(\alpha_{\mathbf{n}} W_{\mathbf{n}, i}\right)(x)} .
$$

Using (22) we obtain

$$
\begin{gathered}
{\left[\frac{Q_{\mathbf{n}} \widehat{s}_{i}-\widehat{P}_{i}}{\alpha_{\mathbf{n}} W_{\mathbf{n}, i}}\right](z)=\int_{\Delta_{1}} \frac{Q_{\mathbf{n}}(x)}{W_{\mathbf{n}, i}(x)} \frac{d s_{i}(x)}{(z-x) \alpha_{\mathbf{n}}(x)}=} \\
\frac{1}{q(z)} \int_{\Delta_{1}} \frac{\left(q Q_{\mathbf{n}}\right)(x)}{\left(\alpha_{\mathbf{n}} W_{\mathbf{n}, i}\right)(x)} \frac{d s_{i}(x)}{z-x}=\mathcal{O}\left(\frac{1}{z^{N^{i}+1}}\right) \in H(D), \quad i=1, \ldots, m .
\end{gathered}
$$

Substracting (23) from (21), we have that

$$
\left[\frac{P_{\mathbf{n}, i}-\widehat{P}_{\mathbf{n}, i}}{\alpha_{\mathbf{n}} W_{\mathbf{n}, i}}\right](z)=\mathcal{O}\left(\frac{1}{z^{N^{i}+1}}\right) \in H(D), \quad i=1, \ldots, m .
$$

Then $\operatorname{deg}\left(P_{\mathbf{n}, i}-\widehat{P}_{\mathbf{n}, i}\right)<\operatorname{deg}\left(\alpha_{\mathbf{n}} W_{\mathbf{n}, i}\right)$ and the polynomial of the numerator vanishes at all the zeros of $\alpha_{\mathbf{n}} W_{\mathbf{n}, i}$ counting multiplicities. Obviously, this implies that $P_{\mathbf{n}, i} \equiv \widehat{P}_{i}$, and we conclude the proof.

Set $N=\max \left\{N^{i}: i=1, \ldots, m\right\}$. From (18) it is obvious that $Q_{\mathbf{n}}$ changes sign at least $N$ times in the interior of $\Delta_{1}$. Hence, $Q_{\mathbf{n}}$ can be represented as the product of two monic polynomials

$$
Q_{\mathbf{n}}=Q_{\mathbf{n}, 1} Q_{\mathbf{n}, 2}, \quad \operatorname{deg} Q_{\mathbf{n}, 1}=\tilde{N} \geq N,
$$

described as follows. The zeros $\left\{x_{\mathbf{n}, j}\right\}, j=1, \ldots, \widetilde{N}$, of the polynomial $Q_{\mathbf{n}, 1}$ are simple and lie in the interior of $\Delta_{1}$. The polynomial $Q_{\mathbf{n}, 2}$ does not change sign on $\Delta_{1}$ and $\operatorname{deg} Q_{\mathbf{n}, 2} \leq|\mathbf{n}|-\widetilde{N}$.

Lemma 3. Fix $i \in\{1, \ldots, m\}$ and $\mathbf{n} \in \mathbb{Z}_{+}^{m}$. Then, for any polynomial $\mathcal{P}, \operatorname{deg} \mathcal{P}<\widetilde{N}+N^{i}$, we have

$$
\int_{\Delta_{1}} \mathcal{P}(x) \frac{Q_{\mathbf{n}, 2}(x) d s_{i}(x)}{\left(\alpha_{\mathbf{n}} W_{\mathbf{n}, i}\right)(x)}=\sum_{j=1}^{\widetilde{N}} \lambda_{\mathbf{n}, j}^{i} \mathcal{P}\left(x_{\mathbf{n}, j}\right)
$$

where

$$
\lambda_{\mathbf{n}, j}^{i}=\int_{\Delta_{1}} \frac{Q_{\mathbf{n}, 1}(x)}{Q_{\mathbf{n}, 1}^{\prime}\left(x_{\mathbf{n}, j}\right)} \frac{Q_{\mathbf{n}, 2}(x) d s_{i}(x)}{\left(x-x_{\mathbf{n}, j}\right)\left(\alpha_{\mathbf{n}} W_{\mathbf{n}, i}\right)(x)} .
$$

Furthermore, for each $i \in\{1, \ldots, m\}$, the number of coefficients $\lambda_{\mathbf{n}, j}^{i}$ whose sign equals that of the measure $\left(Q_{\mathbf{n}, 2} / \alpha_{\mathbf{n}} W_{\mathbf{n}, i}\right)(x) d s_{i}(x)$ is greater than or equal to $\left(\tilde{N}+N^{i}\right) / 2 \geq\left|\mathbf{n}^{i}\right|+n_{i}$. Finally, for any polynomial $q$, $\operatorname{deg} q \leq N^{i}$,

$$
\begin{gathered}
\frac{Q_{\mathbf{n}, 2}(z)\left(\widehat{s}_{i}-\frac{P_{\mathbf{n}, i}}{Q_{\mathbf{n}}}\right)(z)}{\left(\alpha_{\mathbf{n}} W_{\mathbf{n}, i}\right)(z)}=\int_{\Delta_{1}} \frac{\left(Q_{\mathbf{n}, 1} q\right)(x)}{\left(Q_{\mathbf{n}, 1} q\right)(z)} \frac{Q_{\mathbf{n}, 2}(x) d s_{i}(x)}{\left(\alpha_{\mathbf{n}} W_{\mathbf{n}, i}\right)(x)(z-x)} \\
=\int_{\Delta_{1}} \frac{Q_{\mathbf{n}, 2}(x) d s_{i}(x)}{\left(\alpha_{\mathbf{n}} W_{\mathbf{n}, i}\right)(x)(z-x)}-\frac{p_{i}(z)}{Q_{\mathbf{n}, 1}(z)},
\end{gathered}
$$


where

$$
p_{\mathbf{n}, i}=\int_{\Delta_{1}} \frac{Q_{\mathbf{n}, 1}(z)-Q_{\mathbf{n}, 1}(x)}{z-x} \frac{Q_{\mathbf{n}, 2}(x) d s_{i}(x)}{\left(\alpha_{\mathbf{n}} W_{\mathbf{n}, i}\right)(x)} .
$$

and

$$
\frac{p_{\mathbf{n}, i}(z)}{Q_{\mathbf{n}, 1}(z)}=\sum_{j=1}^{\tilde{N}} \frac{\lambda_{\mathbf{n}, j}^{i}}{z-x_{\mathbf{n}, j}} .
$$

Proof. Let $\mathcal{L}$ be the Lagrange polynomial which interpolates $\mathcal{P}$ at each of the points $x_{\mathbf{n}, j}$, $j=1, \ldots, \widetilde{N}$. Then,

$$
\mathcal{P}-\mathcal{L}=Q_{\mathbf{n}, 1} \mathcal{P}_{1}, \quad \operatorname{deg} \mathcal{P}_{1}<N^{i} .
$$

Integrating with respect to the measure $\left.\left(Q_{\mathbf{n}, 2} / \alpha_{\mathbf{n}} W_{\mathbf{n}, i}\right)\right)(x) d s_{i}(x)$ and using (18), we arrive to (24)-(25).

Take

$$
\mathcal{P}(z)=\prod^{+}\left(z-x_{\mathbf{n}, j}\right)^{2},
$$

where $\prod^{+}$denotes the product over all $j$ for which $\lambda_{\mathbf{n}, j}^{i}$ has the same sign as $Q_{\mathbf{n}, 2} /\left(\alpha_{\mathbf{n}} W_{\mathbf{n}, i}\right) d s_{i}$. If $\operatorname{deg} \mathcal{P}<\widetilde{N}+N^{i}$, we can substitute $\mathcal{P}$ into (24) to obtain

$$
\int_{\Delta_{1}} \mathcal{P}(x) \frac{Q_{\mathbf{n}, 2}(x) d s_{i}(x)}{\left(\alpha_{\mathbf{n}} W_{\mathbf{n}, i}\right)(x)}=\sum_{j=1}^{\tilde{N}} \lambda_{\mathbf{n}, j}^{i} \mathcal{P}\left(x_{\mathbf{n}, j}\right) .
$$

Since $\mathcal{P}$ has positive sign on $\Delta_{1}$, the integral on the left hand side has the same sign as the measure $\left.\left(Q_{\mathbf{n}, 2} / \alpha_{\mathbf{n}} W_{\mathbf{n}, i}\right)\right)(x) d s_{i}(x)$. On the right hand side, all the terms corresponding to $\lambda_{\mathbf{n}, j}^{i}$ with the same sign as the measure $\left.\left(Q_{\mathbf{n}, 2} / \alpha_{\mathbf{n}} W_{\mathbf{n}, i}\right)\right)(x) d s_{i}(x)$ vanish. Hence, this sum has a sign different to that of the measure $\left.\left(Q_{\mathbf{n}, 2} / \alpha_{\mathbf{n}} W_{\mathbf{n}, i}\right)\right)(x) d s_{i}(x)$ (or equals zero). This contradiction implies that $\operatorname{deg} \mathcal{P} \geq \widetilde{N}+N^{i}$ and from the definition of $\mathcal{P}$, we obtain the assertion concerning the sign of the coefficients $\lambda_{\mathbf{n}, j}^{i}$.

To prove (26) we use (19), which can be rewriten as

$$
\frac{Q_{\mathbf{n}, 2}(z)\left(\hat{s}_{i}-\frac{P_{\mathbf{n}, i}}{Q_{\mathbf{n}}}\right)(z)}{\left(\alpha_{\mathbf{n}} W_{\mathbf{n}, i}\right)(z)}=\int_{\Delta_{1}} \frac{\left(Q_{\mathbf{n}, 1} q\right)(x)}{\left(Q_{\mathbf{n}, 1} q\right)(z)} \frac{Q_{\mathbf{n}, 2}(x) d s_{i}(x)}{(z-x)\left(\alpha_{\mathbf{n}} W_{\mathbf{n}, i}\right)(x)},
$$

where $q$ is an arbitrary polynomial such that $\operatorname{deg} q \leq N^{i}$. In particular, when $q \equiv 1$ we have that

$$
\begin{aligned}
\frac{Q_{\mathbf{n}, 2}(z)\left(\hat{s}_{i}-\frac{P_{\mathbf{n}, i}}{Q_{\mathbf{n}}}\right)(z)}{\left(\alpha_{\mathbf{n}} W_{\mathbf{n}, i}\right)(z)}= & \int_{\Delta_{1}} \frac{\left(Q_{\mathbf{n}, 1}\right)(x)}{\left(Q_{\mathbf{n}, 1}\right)(z)} \frac{Q_{\mathbf{n}, 2}(x) d s_{i}(x)}{(z-x)\left(\alpha_{\mathbf{n}} W_{\mathbf{n}, i}\right)(x)} \mp \int_{\Delta_{1}} \frac{Q_{\mathbf{n}, 2}(x) d s_{i}(x)}{(z-x)\left(\alpha_{\mathbf{n}} W_{\mathbf{n}, i}\right)(x)}= \\
& \int_{\Delta_{1}} \frac{Q_{\mathbf{n}, 2}(x) d s_{i}(x)}{(z-x)\left(\alpha_{\mathbf{n}} W_{\mathbf{n}, i}\right)(x)}-\frac{p_{\mathbf{n}, i}(z)}{Q_{\mathbf{n}, 1}(z)} .
\end{aligned}
$$

Hence, (26) holds.

Now, since $p_{\mathbf{n}, i}<\operatorname{deg} Q_{\mathbf{n}, 1}$, taking into account that the zeros of $Q_{\mathbf{n}, 1}$ are simple, using (25) and the definition of $p_{\mathbf{n}, i}$, we obtain that

$$
\begin{gathered}
\operatorname{res}\left(\frac{p_{\mathbf{n}, i}}{Q_{\mathbf{n}, 1}}, x_{\mathbf{n}, j}\right)=\lim _{z \rightarrow x_{\mathbf{n}, j}}\left(z-x_{\mathbf{n}, j}\right) \frac{p_{\mathbf{n}, i}(z)}{Q_{\mathbf{n}, 1}(z)} \\
=\lim _{z \rightarrow x_{\mathbf{n}, j}} \frac{z-x_{\mathbf{n}, j}}{Q_{\mathbf{n}, 1}(z)} \int_{\Delta_{1}} \frac{Q_{\mathbf{n}, 1}(z)-Q_{\mathbf{n}, 1}(x)}{z-x} \frac{Q_{\mathbf{n}, 2}(x) d s_{i}(x)}{\left(\alpha_{\mathbf{n}} W_{\mathbf{n}, i}\right)(x)}=\lambda_{\mathbf{n}, j}^{i} .
\end{gathered}
$$

Thus, we have (27) and we finished the proof. 


\section{Proof of Theorem 1}

Proof of Theorem 1. Without loss of generality we can assume that $Q_{\mathbf{n}, 2} d s_{i} /\left(\alpha_{\mathbf{n}} W_{\mathbf{n}, i}\right)$ is a positive measure. Fix $i \in\{1, \ldots, m\}$. We denote

$$
g_{\mathbf{n}}(z)=\bar{\prod}\left(z-x_{\mathbf{n}, j}\right)^{2}
$$

where $\Pi^{-}$denotes the product over all the zeros of $Q_{\mathbf{n}, 1}$ such that $\lambda_{\mathbf{n}, j}^{i}<0$. From Lemma 3 and the assumptions, we have that

$$
\operatorname{deg} g_{\mathbf{n}} \leq 2\left(\tilde{N}-\frac{\tilde{N}+N^{i}}{2}\right)=\tilde{N}-N^{i} \leq|\mathbf{n}|-\left|\mathbf{n}^{i}\right|-n_{i} \leq c m|\mathbf{n}|^{\kappa} .
$$

and

$$
\operatorname{deg} Q_{\mathbf{n}, 2}=|\mathbf{n}|-\tilde{N} \leq|\mathbf{n}|-\left|\mathbf{n}^{i}\right|-n_{i} \leq c m|\mathbf{n}|^{\kappa} .
$$

Let $\widetilde{W}_{\mathbf{n}}$ be a monic polynomial with real coefficients that divides $\alpha_{\mathbf{n}} W_{\mathbf{n}, i}$. We take $\widetilde{W}_{\mathbf{n}}$ to be of degree sufficiently large so as to able to use the quadrature formula (24) on the polynomial

$$
\mathcal{P}=p_{\mathbf{n}}=\frac{g_{\mathbf{n}} \alpha_{\mathbf{n}} W_{\mathbf{n}, i}}{\widetilde{W}_{\mathbf{n}}}
$$

That is, we want that

$$
\operatorname{deg} p_{\mathbf{n}}<\operatorname{deg} Q_{\mathbf{n}, 1}+\left|\mathbf{n}^{i}\right|+n_{i} .
$$

Additionaly, it is convenient that the degree of $p_{\mathbf{n}}$ be as large as possible. We will see later the advantage of this. It is sufficient to take $\widetilde{W}_{\mathbf{n}}$ such that

$$
3 c m|\mathbf{n}|^{\kappa}<\operatorname{deg} \widetilde{W_{\mathbf{n}}} \leq 3 \mathrm{~cm}|\mathbf{n}|^{\kappa}+2
$$

In fact, using this restriction and (3), we have that

$\operatorname{deg} p_{\mathbf{n}}=\operatorname{deg} g_{\mathbf{n}}+\operatorname{deg} \alpha_{\mathbf{n}}+\operatorname{deg} W_{\mathbf{n}, i}-\operatorname{deg} \widetilde{W}_{\mathbf{n}}<2\left(|\mathbf{n}|-c m|n|^{\kappa}\right) \leq 2\left(\left|\mathbf{n}^{i}\right|+n_{i}\right) \leq \operatorname{deg} Q_{\mathbf{n}, 1}+\left|\mathbf{n}^{i}\right|+n_{i}$ as needed. The extra 2 in the upper bound of $\operatorname{deg} \widetilde{W}_{\mathbf{n}}$ is to be able to select conjugate pairs of zeros, if needed, to guarantee that $\widetilde{W}_{\mathbf{n}}$ has real coefficients.

Let $M=2 \max \left\{1, \max \left\{|z|: z \in \Delta_{1}\right\}\right\}$. We denote by $B_{\mathbf{n}}$ the product of the zeros of $Q_{\mathbf{n}, 2}$ whose absolute value is larger than $M$, counting multiplicities. According to the upper bound obtained for the degree of $Q_{\mathbf{n}, 2}$, this polynomial has at most $\mathrm{cm}|\mathbf{n}|^{\kappa}$ zeros of absolute value greater than $M$.

Consider the function

$$
K_{\mathbf{n}}(x, z)=\frac{p_{\mathbf{n}}(z)-p_{\mathbf{n}}(x)}{(z-x) p_{\mathbf{n}}(z)} .
$$

This is a polynomial on $x$ of degree $<\operatorname{deg} Q_{\mathbf{n}, 1}+\left|\mathbf{n}^{i}\right|+n_{i}$. Hence, appling the quadrature formula (24) and taking into account (24), (26), and (27), we have

$$
\begin{gathered}
\Omega_{\mathbf{n}}(z)=\frac{g_{\mathbf{n}} Q_{\mathbf{n}, 2}\left(\widehat{s}_{i}-R_{\mathbf{n}, i}\right)}{B_{\mathbf{n}} \widetilde{W}_{\mathbf{n}}}=\frac{\left(g_{\mathbf{n}} \alpha_{\mathbf{n}} W_{\mathbf{n}, i}\right)(z)}{B_{\mathbf{n}} \widetilde{W}_{\mathbf{n}}(z)}\left[\int_{\Delta_{1}} \frac{1}{z-x} \frac{Q_{\mathbf{n}, 2}(x) d s_{i}(x)}{\left(\alpha_{\mathbf{n}} W_{\mathbf{n}, i}\right)(x)}-\frac{p_{\mathbf{n}, i}(z)}{Q_{\mathbf{n}, 1}(z)}\right]= \\
\frac{\left(g_{\mathbf{n}} \alpha_{\mathbf{n}} W_{\mathbf{n}, i}\right)(z)}{B_{\mathbf{n}} \widetilde{W}_{\mathbf{n}}(z)}\left[\int_{\Delta_{1}}\left(\frac{1}{z-x}-K_{\mathbf{n}}(x, z)\right) \frac{Q_{\mathbf{n}, 2}(x) d s_{i}(x)}{\left(\alpha_{\mathbf{n}} W_{\mathbf{n}, i}\right)(x)}-\right. \\
\frac{\left.\sum_{j=1}^{\operatorname{deg} Q_{\mathbf{n}, 1}} \lambda_{\mathbf{n}, j}^{i}\left(\frac{1}{z-x_{\mathbf{n}, j}}-K_{\mathbf{n}}\left(x_{\mathbf{n}, j}, z\right)\right)\right]=}{\frac{\left(g_{\mathbf{n}} \alpha_{\mathbf{n}} W_{\mathbf{n}, i}\right)(z)}{B_{\mathbf{n}} \widetilde{W}_{\mathbf{n}}(z)}\left[\int_{\Delta_{1}} \frac{p_{\mathbf{n}}(x)}{(z-x) p_{\mathbf{n}}(z)} \frac{Q_{\mathbf{n}, 2}(x) d s_{i}(x)}{\left(\alpha_{\mathbf{n}} W_{\mathbf{n}, i}\right)(x)}-\sum_{j=1}^{\operatorname{deg} Q_{\mathbf{n}, 1}} \lambda_{\mathbf{n}, j}^{i} \frac{p_{\mathbf{n}}\left(x_{\mathbf{n}, j}\right)}{\left(z-x_{\mathbf{n}, j}\right) p_{\mathbf{n}}(z)}\right]} \\
=\frac{1}{B_{\mathbf{n}}}\left[\int_{\Delta_{1}} \frac{p_{\mathbf{n}}(x)}{z-x} \frac{Q_{\mathbf{n}, 2}(x) d s_{i}(x)}{\left(\alpha_{\mathbf{n}} W_{\mathbf{n}, i}\right)(x)}-\sum_{j=1}^{\operatorname{deg} Q_{\mathbf{n}, 1}} \lambda_{\mathbf{n}, j}^{i} \frac{p_{\mathbf{n}}\left(x_{\mathbf{n}, j}\right)}{z-x_{\mathbf{n}, j}}\right]
\end{gathered}
$$


Denote by $d\left(z, \Delta_{1}\right)$ the distance between $z$ and $\Delta_{1}$. Using (24) once more, we obtain that

$$
\begin{gathered}
\left|\sum_{j=1}^{\operatorname{deg} Q_{\mathbf{n}, 1}} \lambda_{\mathbf{n}, j}^{i} \frac{p_{\mathbf{n}}\left(x_{\mathbf{n}, j}\right)}{z-x_{\mathbf{n}, j}}\right| \leq \frac{1}{d\left(z, \Delta_{1}\right)} \sum_{j=1}^{\operatorname{deg} Q_{\mathbf{n}, 1}}\left|\lambda_{\mathbf{n}, j}^{i} p_{\mathbf{n}}\left(x_{\mathbf{n}, j}\right)\right|= \\
\frac{1}{d\left(z, \Delta_{1}\right)}\left|\int p_{\mathbf{n}}(x) \frac{Q_{\mathbf{n}, 2}(x) d s_{i}(x)}{\left(\alpha_{\mathbf{n}} W_{\mathbf{n}, i}\right)(x)}\right| .
\end{gathered}
$$

When we apply the quadrature formula, we use that all the terms of the sum have the same sign as $Q_{\mathbf{n}, 2}(x) d s_{i}(x) /\left(\alpha_{\mathbf{n}} W_{\mathbf{n}, i}\right)(x)$ or are equal to zero since this polynomial $p_{\mathbf{n}}$ vanishes at all the zeros of $g_{\mathbf{n}}$ on $\Delta_{1}$ and $\alpha_{\mathbf{n}} W_{\mathbf{n}, i} / \widetilde{W}_{\mathbf{n}}$ is a polynomial whose coefficients are real numbers and its zeros lie outside of the interval $\Delta_{1}$. Reasoning analogously, we obtain that

$$
\left|\int \frac{p_{\mathbf{n}}(x)}{z-x} \frac{Q_{\mathbf{n}, 2}(x) d s_{i}(x)}{\left(\alpha_{\mathbf{n}} W_{\mathbf{n}, i}\right)(x)}\right| \leq \frac{1}{d\left(z, \Delta_{1}\right)}\left|\int p_{\mathbf{n}}(x) \frac{Q_{\mathbf{n}, 2}(x) d s_{i}(x)}{\left(\alpha_{\mathbf{n}} W_{\mathbf{n}, i}\right)(x)}\right| .
$$

Therefore,

$$
\begin{gathered}
\left|\Omega_{\mathbf{n}}(z)\right| \leq\left|\frac{2}{B_{\mathbf{n}} d\left(z, \Delta_{1}\right)} \int_{\Delta_{1}} p_{\mathbf{n}}(x) \frac{Q_{\mathbf{n}, 2}(x) d s_{i}(x)}{\left(\alpha_{\mathbf{n}} W_{\mathbf{n}, i}\right)(x)}\right|= \\
\left|\frac{2}{B_{\mathbf{n}} d\left(z, \Delta_{1}\right)}\right|\left|\int_{\Delta_{1}}\left(g_{\mathbf{n}} Q_{\mathbf{n}, 2}\right)(x) \frac{d s_{i}(x)}{\widetilde{W}_{\mathbf{n}}(x)}\right| \leq \frac{2\left|s_{i}\right|}{d\left(z, \Delta_{1}\right)}\left\|\frac{g_{\mathbf{n}} Q_{\mathbf{n}, 2}}{B_{\mathbf{n}} \widetilde{W}_{\mathbf{n}}}\right\|_{\Delta_{1}} .
\end{gathered}
$$

To estimate $\left\|g_{\mathbf{n}} Q_{\mathbf{n}, 2} / B_{\mathbf{n}} \widetilde{W}_{\mathbf{n}}\right\|_{\Delta_{1}}$, we analyze each factor separately. The zeros of $g_{\mathbf{n}}$ lie on $\Delta_{1}$, hence

$$
\left\|g_{\mathbf{n}}\right\|_{\Delta_{1}} \leq\left(2\|x\|_{\Delta_{1}}\right)^{\operatorname{deg} g_{\mathbf{n}}} \leq M^{c m|\mathbf{n}|^{\kappa}} .
$$

The zeros of $\widetilde{W}_{\mathbf{n}}$ are on $\Delta_{2} \cup F$. Set $d=\min \left\{1, d\left(\Delta_{1}, \Delta_{2} \cup F\right)\right\}$. Obviously, $d>0$. Then

$$
\left\|\frac{1}{\widetilde{W}_{\mathbf{n}}}\right\|_{\Delta_{1}} \leq\left(\frac{1}{d}\right)^{\operatorname{deg} \widetilde{W}_{\mathbf{n}}} \leq\left(\frac{M}{d}\right)^{4 c m|\mathbf{n}|^{\kappa}} .
$$

To find a bound for the values of $Q_{\mathbf{n}, 2} / B_{\mathbf{n}}$, we consider each one of its zeros. Let $\zeta$ be a zero of $Q_{\mathbf{n}, 2}$. If $|\zeta| \leq M$, we have that

$$
\|x-\zeta\|_{\Delta_{1}} \leq\|x\|_{\Delta_{1}}+|\zeta| \leq 2 M .
$$

On other hand, if $|\zeta|>M, B_{\mathbf{n}}$ includes $\zeta$ as a one of its factors, and

$$
\left\|\frac{x-\zeta}{\zeta}\right\|_{\Delta_{1}} \leq 1+|\zeta|^{-1}\|x\|_{\Delta_{1}} \leq 2 M
$$

Therefore,

$$
\left\|\frac{Q_{\mathbf{n}, 2}}{B_{\mathbf{n}}}\right\|_{\Delta_{1}} \leq(2 M)^{\operatorname{deg} Q_{\mathbf{n}, 2}} \leq M^{2 c m|\mathbf{n}|^{\kappa}} .
$$

Finally, we arrive to the following estimate

$$
\left\|\frac{g_{\mathbf{n}} Q_{\mathbf{n}, 2}}{B_{\mathbf{n}} \widetilde{W}_{\mathbf{n}}}\right\|_{\Delta_{1}} \leq\left(\frac{M}{d}\right)^{4 c m|\mathbf{n}|^{\kappa}}
$$

which together with (28), yields

$$
\left|\Omega_{\mathbf{n}}(z)\right| \leq \frac{2\left|s_{i}\right|}{d\left(z, \Delta_{1}\right)}\left(\frac{M}{d}\right)^{4 c m|\mathbf{n}|^{\kappa}}, \quad z \in \overline{\mathbb{C}} \backslash \Delta_{1} .
$$

That is

$$
\frac{g_{\mathbf{n}} Q_{\mathbf{n}, 2}\left(\widehat{s}_{i}-R_{\mathbf{n}, i}\right)}{B_{\mathbf{n}} \widetilde{W}_{\mathbf{n}}}=\mathcal{O}\left(\frac{1}{z}\right) \in H\left(\overline{\mathbb{C}} \backslash \Delta_{1}\right), \quad z \rightarrow \infty .
$$

and on each compact subset $K \subset D=\overline{\mathbb{C}} \backslash \Delta_{1}$ we have that

$$
\left\|\frac{g_{\mathbf{n}} Q_{\mathbf{n}, 2}\left(\widehat{s}_{i}-R_{\mathbf{n}, i}\right)}{B_{\mathbf{n}} \widetilde{W}_{\mathbf{n}}}\right\|_{K} \leq \frac{2\left|s_{i}\right|}{d\left(K, \Delta_{1}\right)}\left(\frac{M}{d}\right)^{4 c m|\mathbf{n}|^{\kappa}}
$$


Take $\varphi_{t}$ as defined in the statement of the Theorem. Set $\gamma_{\rho}=\left\{z:\left|\varphi_{\infty}(z)\right|=\rho\right\}, 0<\rho<1$. From (29), we have that

$$
\left\|\frac{g_{\mathbf{n}} Q_{\mathbf{n}, 2}\left(\widehat{s}_{i}-R_{\mathbf{n}, i}\right)}{B_{\mathbf{n}} \widetilde{W}_{\mathbf{n}}}\right\|_{\gamma_{\rho}} \leq \frac{2\left|s_{i}\right|}{d\left(\gamma_{\rho}, \Delta_{1}\right)}\left(\frac{M}{d}\right)^{4 c m|\mathbf{n}|^{\kappa}} .
$$

Fix a compact set $K \subset D$. Take $\rho$ sufficiently close to 1 so that $F \cup \Delta_{2} \cup K \subset \operatorname{Ext}\left(\gamma_{\rho}\right)$. Set $v_{\mathbf{n}}=$ $\operatorname{deg}\left(\alpha_{\mathbf{n}} W_{\mathbf{n}, i} / \widetilde{W}_{\mathbf{n}}\right)$. Let $w_{\mathbf{n}}$ be the exact multiplicity of the zero which $\Omega_{\mathbf{n}}$ has at infinity. Taking into account that $\operatorname{deg}\left(g_{\mathbf{n}} Q_{\mathbf{n}, 2}\right) \leq 2 \mathrm{~cm}|\mathbf{n}|^{\kappa}, \operatorname{deg} Q_{\mathbf{n}, 1} \geq\left|\mathbf{n}^{i}\right|+n_{i} \geq|\mathbf{n}|-c m|\mathbf{n}|^{\kappa}, \operatorname{deg} \widetilde{W}_{\mathbf{n}} \leq 4 \mathrm{~cm}|\mathbf{n}|^{\kappa}$ and $\operatorname{deg} W_{\mathbf{n}, i} \geq(m-1)\left(\frac{|\mathbf{n}|}{m}-c|\mathbf{n}|^{\kappa}\right)$, from ii) in (2), we have that there exists a constant $c^{\prime}>0$ such that

$$
2|\mathbf{n}|-c^{\prime}|\mathbf{n}|^{\kappa} \leq w_{\mathbf{n}}+v_{\mathbf{n}} \leq 2|\mathbf{n}| .
$$

Let $\left\{y_{\mathbf{n}, 1}, \ldots, y_{\mathbf{n}, v_{\mathbf{n}}}\right\}$ be the set of zeros of the polynomial $\left(\alpha_{\mathbf{n}} W_{\mathbf{n}} / \widetilde{W}_{\mathbf{n}}\right)$. We have that

$$
\frac{g_{\mathbf{n}} Q_{\mathbf{n}, 2}\left(\widehat{s}_{i}-R_{\mathbf{n}, i}\right)}{B_{\mathbf{n}} \widetilde{W}_{\mathbf{n}} \varphi_{\infty}^{\omega_{\mathbf{n}}} \prod_{j=1}^{v_{\mathbf{n}}} \varphi_{y_{\mathbf{n}, j}}} \in H(D)
$$

Define

$$
\kappa\left(\gamma_{\rho}\right)=\inf \left\{\left|\varphi_{t}(z)\right|: z \in \gamma_{\rho}, t \in F \cup \Delta_{2} \cup\{\infty\}\right\}>0 .
$$

As a function of the two variables $z$ and $t,\left|\varphi_{t}(z)\right|$ is continuous on $\overline{\mathbb{C}}^{2}$. Hence $\kappa\left(\gamma_{\rho}\right)>0$, because $\gamma_{\rho} \cap\left(F \cup \Delta_{2}\right)=\emptyset$ and $\left|\varphi_{t}(z)\right|$ vanishes only when $z=t$.

From (30), we obtain that

$$
\left\|\frac{g_{\mathbf{n}} Q_{\mathbf{n}, 2}\left(\widehat{s}_{i}-R_{\mathbf{n}, i}\right)}{B_{\mathbf{n}} \widetilde{W}_{\mathbf{n}} \varphi_{\infty}^{\omega_{\mathbf{n}}} \prod_{j=1}^{v_{\mathbf{n}}} \varphi_{y_{\mathbf{n}, j}}}\right\|_{\gamma_{\rho}} \leq \frac{2\left|s_{i}\right|}{d\left(\gamma_{\rho}, \Delta_{1}\right) \kappa\left(\gamma_{\rho}\right)^{w_{\mathbf{n}}+v_{\mathbf{n}}}}\left(\frac{M}{d}\right)^{4 c m|\mathbf{n}|^{\kappa}} .
$$

Since $K \subset \operatorname{Ext}\left(\gamma_{\rho}\right)$, using the maximum principle for analytic functions, we have that this inequality holds for all $z \in K$. Then

$$
\begin{gathered}
\left|\frac{g_{\mathbf{n}} Q_{\mathbf{n}, 2}\left(\widehat{s}_{i}-R_{\mathbf{n}, i}\right)}{B_{\mathbf{n}} \widetilde{W}_{\mathbf{n}}}\right| \leq \\
\frac{2\left|s_{i}\right|}{d\left(\gamma_{\rho}, \Delta_{1}\right) \kappa\left(\gamma_{\rho}\right)^{w_{\mathbf{n}}+v_{\mathbf{n}}}}\left(\frac{M}{d}\right)^{4 c m|\mathbf{n}|^{\kappa}}\left|\varphi_{\infty}^{\omega_{\mathbf{n}}}(z) \prod_{j=1}^{v_{\mathbf{n}}} \varphi_{y_{\mathbf{n}, j}}(z)\right|, \quad z \in K .
\end{gathered}
$$

Set

$$
\delta_{K}=\max \left\{\left|\varphi_{t}(z)\right|: z \in K, t \in F \cup \Delta_{2} \cup\{\infty\}\right\}<1 .
$$

By the continuity of $\left|\varphi_{t}(z)\right|$ on $\overline{\mathbb{C}}^{2}$, we can assure that $\delta_{K}<1$, because it is equal to 1 only when $z \in \Delta_{1}$. From the definition of $\delta_{K}$, the last inequality, and (31), we obtain that

$$
\underset{\mathbf{n} \in \Lambda}{\limsup }\left\|\frac{g_{\mathbf{n}} Q_{\mathbf{n}, 2}\left(\widehat{s}_{i}-R_{\mathbf{n}, i}\right)}{B_{\mathbf{n}} \widetilde{W}_{\mathbf{n}}}\right\|_{K}^{1 / 2|\mathbf{n}|} \leq \frac{\delta_{K}}{\kappa\left(\gamma_{\rho}\right)} .
$$

Making $\rho$ tend to 1 , we have that $\kappa\left(\gamma_{\rho}\right)$ tends to 1 ; hence, we conclude that

$$
\limsup _{\mathbf{n} \in \Lambda}\left\|\Omega_{\mathbf{n}}\right\|_{K}^{1 / 2|\mathbf{n}|}=\limsup _{\mathbf{n} \in \Lambda}\left\|\frac{g_{\mathbf{n}} Q_{\mathbf{n}, 2}\left(\widehat{s}_{i}-R_{\mathbf{n}, i}\right)}{B_{\mathbf{n}} \widetilde{W}_{\mathbf{n}}}\right\|_{K}^{1 / 2|\mathbf{n}|} \leq \delta_{K}<1 .
$$

In particular, for each compact $K \subset D$ and $\delta>0$,

$$
\left|\Omega_{\mathbf{n}}(z)\right| \leq\left(\delta_{K}+\delta\right)^{2|\mathbf{n}|}, \quad z \in K
$$

for all $\mathbf{n} \in \Lambda$, except a finite number of multi-indices.

Notice that

$$
\widehat{s}_{i}-R_{\mathbf{n}, i}=\frac{B_{\mathbf{n}} \widetilde{W}_{\mathbf{n}} \Omega_{\mathbf{n}}}{g_{\mathbf{n}} Q_{\mathbf{n}, 2}} .
$$

Set $R=2 \max \left\{\|z\|_{K}, 1\right\}$. Let $Q_{\mathbf{n}, R}$ be the monic polynomial whose zeros are those of $Q_{\mathbf{n}, 2}$ with absolute value less than $R$. Let us obtain an estimate of $\left\|\left(B_{\mathbf{n}} \widetilde{W}_{\mathbf{n}} Q_{\mathbf{n}, R}\right) /\left(g_{\mathbf{n}} Q_{\mathbf{n}, 2}\right)\right\|_{K}$. 
Since the zeros of $g_{\mathbf{n}}$ lie in $\Delta_{1}$, then

$$
\left\|\frac{1}{g_{\mathbf{n}}}\right\|_{K} \leq\left(\frac{1}{d\left(K, \Delta_{1}\right)}\right)^{\operatorname{deg} g_{\mathbf{n}}} \leq\left(\frac{1}{d_{1}}\right)^{c m|\mathbf{n}|^{\kappa}},
$$

where $d_{1}=\min \left\{d\left(K, \Delta_{1}\right), 1\right\}$. The zeros of $\widetilde{W}_{\mathbf{n}}$ lie on $F \cup \Delta_{2}$, thus

$$
\left\|\widetilde{W}_{\mathbf{n}}\right\|_{K} \leq\left(M\left(K, F \cup \Delta_{2}\right)\right)^{\operatorname{deg} \widetilde{W}_{\mathbf{n}}} \leq M_{1}^{4 c m|\mathbf{n}|^{\kappa}},
$$

where $M\left(K, F \cup \Delta_{2}\right)=\max \left\{|z-\zeta|: z \in K, \zeta \in F \cup \Delta_{2}\right\}$ and $M_{1}=\max \left\{M\left(K, F \cup \Delta_{2}\right), 1\right\}$. If $\zeta$ is a zero of $Q_{\mathbf{n}, 2} / Q_{\mathbf{n}, R}$, since $\zeta$ is a factor of $B_{\mathbf{n}}$, we obtain

$$
\left|\frac{\zeta}{z-\zeta}\right|=\frac{1}{\left|1-\frac{z}{\zeta}\right|} \leq \frac{1}{1-\left|\frac{z}{\zeta}\right|} \leq 2 \leq R .
$$

The other factors of $B_{\mathbf{n}}$ have absolute value less than $R$. Hence,

We conclude that

$$
\left\|\frac{B_{\mathbf{n}} Q_{\mathbf{n}, R}}{Q_{\mathbf{n}, 2}}\right\|_{K} \leq R^{c m|\mathbf{n}|^{\kappa}}
$$

$$
\left\|\frac{B_{\mathbf{n}} \widetilde{W}_{\mathbf{n}} Q_{\mathbf{n}, R}}{g_{\mathbf{n}} Q_{\mathbf{n}, 2}}\right\|_{K} \leq\left(\frac{R M_{1}}{d_{1}}\right)^{4 c m|\mathbf{n}|^{\kappa}} .
$$

Let $\delta>0$ be an arbitrary positive number, such that $\delta_{K}+\delta<1$. Using (32), (33) and (34), we obtain

$$
\left|\left(\widehat{s}_{i}-R_{\mathbf{n}, i}\right)(z)\right| \leq \frac{\left(\delta_{K}+\delta\right)^{2|\mathbf{n}|}}{\left|Q_{\mathbf{n}, R}(z)\right|}\left(\frac{R M_{1}}{d_{1}}\right)^{4 c m|\mathbf{n}|^{\kappa}}, \quad z \in K .
$$

In case that there exists an infinite subsequence of multi-indices $\Lambda^{\prime} \subset \Lambda$ such that $Q_{\mathbf{n}, R} \equiv 1, n \in \Lambda^{\prime}$, from the last inequality, we obtain that

$$
\limsup _{\mathbf{n} \in \Lambda^{\prime}}\left\|\widehat{s}_{i}-R_{\mathbf{n}, i}\right\|_{K}^{1 / 2|\mathbf{n}|} \leq \delta_{K}+\delta .
$$

Making $\delta$ tend to 0 , we arrive to

$$
\limsup _{\mathbf{n} \in \Lambda^{\prime}}\left\|\widehat{s}_{i}-R_{\mathbf{n}, i}\right\|_{K}^{1 / 2|\mathbf{n}|} \leq \delta_{K}
$$

This is the case for the whole sequence $\Lambda$ if $m \leq 3$ or $m>3$ and $\Lambda \subset \mathbb{Z}_{+}^{m}(*)$ as in the hypothesis of Corollary 1 as follows from Lemma 2. Thus Corollary 1 is proved. In general, if such a subsequence exists, for that subsequence we have uniform convergence, which is stronger than convergence in Hausdorff content. Therefore, without loss of generality, we can assume $\operatorname{deg} Q_{\mathbf{n}, R} \geq 1, n \in \Lambda$.

Fix $\varepsilon>0$. From (35), it follows that

$$
\begin{gathered}
\left\{z \in K:\left|\left(\widehat{s}_{i}-R_{\mathbf{n}, i}\right)(z)\right|>\epsilon\right\} \subset \\
\left\{z \in K: \frac{\left(\delta_{K}+\delta\right)^{2|\mathbf{n}|}}{\left|Q_{\mathbf{n}, R}(z)\right|}\left(\frac{R M_{1}}{d_{1}}\right)^{4 c m|\mathbf{n}|^{\kappa}}>\varepsilon\right\}= \\
\left\{z \in K:\left|Q_{\mathbf{n}, R}(z)\right|<\frac{\left(\delta_{K}+\delta\right)^{2|\mathbf{n}|}}{\varepsilon}\left(\frac{R M_{1}}{d_{1}}\right)^{4 c m|\mathbf{n}|^{\kappa}}\right\} \subset \\
\left\{z \in \mathbb{C}:\left|Q_{\mathbf{n}, R}(z)\right|<\frac{\left(\delta_{K}+\delta\right)^{2|\mathbf{n}|}}{\varepsilon}\left(\frac{R M_{1}}{d_{1}}\right)^{4 c m|\mathbf{n}|^{\kappa}}\right\} \subset \\
\bigcup_{\left\{\zeta: Q_{\mathbf{n}, R}(\zeta)=0\right\}}\left\{z \in \mathbb{C}:|z-\zeta|<\left(\frac{\left(\delta_{K}+\delta\right)^{2|\mathbf{n}|}}{\varepsilon}\left(\frac{R M_{1}}{d_{1}}\right)^{4 c m|\mathbf{n}|^{\kappa}}\right)^{1 / \operatorname{deg} Q_{\mathbf{n}, R}}\right\} .
\end{gathered}
$$

From the subaditivity and monotonicity of the Hausdorff content, we deduce that

$$
m_{1}\left(\left\{z \in K:\left|\left(\widehat{s}_{i}-R_{\mathbf{n}, i}\right)(z)\right|>\epsilon\right\}\right) \leq \operatorname{deg} Q_{\mathbf{n}, R}\left(\frac{\left(\delta_{K}+\delta\right)^{2|\mathbf{n}|}}{\varepsilon}\left(\frac{R M_{1}}{d_{1}}\right)^{4 c m|\mathbf{n}|^{\kappa}}\right)^{1 / \operatorname{deg} Q_{\mathbf{n}, R}} .
$$


Since $1 \leq \operatorname{deg} Q_{\mathbf{n}, R} \leq \mathrm{cm}|\mathbf{n}|^{\kappa}$, we obtain that

$$
\limsup _{\mathbf{n} \in \Lambda}\left(m_{1}\left(\left\{z \in K:\left|\left(\widehat{s}_{i}-R_{\mathbf{n}, i}\right)(z)\right|>\varepsilon\right\}\right)\right)^{1 / 2|\mathbf{n}|} \leq \delta_{K}+\delta .
$$

Letting $\delta \rightarrow 0$, we have (4). With this we conclude the proof.

REMARK . These results hold true if in the definition of a Nikishin system we only require that the interior (in $\mathbb{R}$ ) of $\Delta_{j} \cap \Delta_{j+1}, j=2, \ldots, m-1$, be empty as long as the measures $s_{j}, j=1, \ldots, m$, are finite. This allows consecutive intervals $\Delta_{j}$ to have a common end point (starting from the second one). If $\Delta_{1}$ and $\Delta_{2}$ have a common end point, we cannot rely on the extra interpolation points which appear on $\Delta_{2}$ to deduce the rate of convergence because they can accumulate on $\Delta_{1}$ and then $\delta_{K}=1$. Disregarding these extra interpolation points and following basically the same scheme of proof, instead of (4) one can prove

$$
\underset{\mathbf{n} \in \Lambda}{\limsup }\left(m_{1}\left(\left\{z \in K:\left|\left(\widehat{s}_{i}-R_{\mathbf{n}, i}\right)(z)\right|>\varepsilon\right\}\right)\right)^{m /|\mathbf{n}|(m+1)} \leq \delta_{K}^{\prime}<1,
$$

where

$$
\delta_{K}^{\prime}=\max \left\{\left|\varphi_{t}(z)\right|: z \in K, t \in F \cup\{\infty\}\right\},
$$

which is sufficient to prove (5), and in place of (6) one gets

$$
\limsup _{\mathbf{n} \in \Lambda}\left\|\widehat{s}_{i}-R_{\mathbf{n}, i}\right\|_{K}^{m /|\mathbf{n}|(m+1)} \leq \delta_{K}^{\prime}<1, \quad i=1, \ldots, m,
$$

from which (7) follows. Certainly, these bounds are also true when $\Delta_{1} \cap \Delta_{2}=\emptyset$. One has to compare $\delta_{K}^{2}$ and $\left(\delta_{K}^{\prime}\right)^{(m+1) / m}$ and choose the smaller one of these values to give a better estimate. For example, if $\Delta_{2}$ is very close to $\Delta_{1}$ the second one of these values may be smaller than the first. Assumption (3) (either when $\Delta_{1}$ and $\Delta_{2}$ are disjoint or have a common end point) can be replaced by

$$
n_{i} \geq \frac{|\mathbf{n}|}{m}-o(|\mathbf{n}|), \quad i=1, \ldots, m,
$$

and Theorem 1 and Corollary 1 remain valid. We have restricted generality in the statements in order to simplify the arguments in the proofs, though basically they remain the same.

REMARK . The measures $\sigma_{j}$ may be allowed to have unbounded support. If $\operatorname{supp}\left(\sigma_{1}\right)$ remains a compact set nothing changes. When $\operatorname{supp}\left(\sigma_{1}\right)$ is an unbounded subset of the real line (say $\Delta_{1}=[0,+\infty)$ ), geometric rate of convergence in (4) and (6) may not take place if $\Delta_{2}$ is also unbounded. Nevertheless, even in this situation one can still prove (5) and (7) assuming, for example, Carleman's condition (see [2])

$$
\sum_{n=0}^{\infty}\left(\frac{1}{c_{n}}\right)^{1 / 2 n}=\infty, \quad c_{n}=\int x^{n} d \sigma_{1}(x) .
$$

In this case the proof is substantially more difficult and additional arguments are required. See [1] to understand the methodology to be used.

\section{REFERENCES}

[1] ZH. Bustamante And G. Lopez Lagomasino. Hermite-Padé approximation for Nikishin systems of analytic functions. Russian Acad. Sci. Sb. Math., 77 (1994), 367-384.

[2] T. Carleman. Les Fonctions Quasi-Analytiques. Gauthier-Villars, Paris, 1926.

[3] K. Driver And H. Stahl. Normality in Nikishin systems. Indag. Math., 5 (1994), 161-187.

[4] K. Driver and H. Stahl. Simultaneous rational approximants to Nikishin systems II. Acta Sci. Math., 61 (1995), 261-284

[5] U. Fidalgo and G. López Lagomasino. On perfect Nikishin systems. Comp. Methods and Function Theory, $2(2002), 415-426$

[6] U. Fidalgo, J. Illán, And G. López Lagomasino.Hermite-Padé approcximants and simultaneous quadraturas formulas. J. of Approx. Theory, 126 (2004), 171-197.

[7] U. Fidalgo and G. López Lagomasino.Rate of convergence of generalized Hermite-Padé approximants of Nikishin systems. Constr. Approx. (in press).

[8] A. A. Gonchar. On the convergence of generalized Padé approximants for meromorphic functions. Math. USSR. Sb., 27 (1975), 503-514.

[9] A. A. Gonchar And G. Lopez Lagomasino. On Markov's theorem for multipoint Padé approximants. Math. USSR Sb. 34 (1978), 449-459. 
[10] M. G. Krein and A. A. Nudel'man. The Markov Moment Problem and Extremal Problems. Transl. of Math. Monographs, Vol 15, Amer. Math. Soc., Providence, 1977.

[11] N. S. LandKof. Foundations of Modern Potential Theory. Grundlehren der Mathenatischen Wissenschaften, Vol. 190, Springer Verlag, New York, 1972.

[12] A. A. Markov. Deux demonstrations de la convergence de certaine fractions continues. Acta Math. 19 (1895), 93-104.

[13] E. M. Nikishin. On simultaneous Padé approximants. Math. USSR Sb., 41 (1982), 409-425.

[14] H. Stahl and V. Tотік, General Orthogonal Polynomials. Cambridge University Press, Cambridge, 1992.

(Ulises) Departamento de Matemáticas, Universidad Carlos III de Madrid, C/ Universidad 30, 28911 LEgAnÉs, SPAin.

E-mail address, Ulises: ulisesfidalgoprieto@yahoo.es

(Guillermo) Departamento de Matemáticas, Universidad Carlos III, C/ Universidad 30, 28911 Leganés, SPAIN.

E-mail address, Guillermo: lago@math.uc3m.es 